\title{
Anestesia espinal. Parte II: Importancia de la anatomía, indicaciones y drogas más usadas
}

\author{
Spinal anesthesia. Part II: The importance of anatomy, \\ indications and drugs
}

Héctor Lacassie Q. ${ }^{1}$, Juan Carlos De La Cuadra F. ${ }^{1}$, Catalina Kychenthal L. ${ }^{2}$, María Jesús Irarrázaval M.2, Fernando R. Altermatt C. ${ }^{1}$

\begin{abstract}
The spinal anaesthesia requires knowledge of the anatomy of the involved parts, as well as its variations with position and the eventual injured structures. Spinal anaesthesia is indicated when the surgical procedure can be accomplished with a sensitive level of anesthesia that does not affect the patients comfort. Absolute contraindications are the patients rejection of the technique, infection at the punction site, sepsis and other forms of circulatory failure, endocranial hypertension and severe coagulopathy. The success of this technique depends in part of the local anesthesic and the most commonly used are lidocaine and bupivacaine.
\end{abstract}

\section{RESUMEN}

El bloqueo espinal requiere de un conocimiento acabado de la anatomía de las partes involucradas, así como de sus variaciones con la posición y posibles estructuras a lesionar. La anestesia espinal está indicada cuando el procedimiento quirúrgico puede lograrse con un nivel sensitivo de anestesia que no afecte el bienestar del paciente. Sus contraindicaciones absolutas son el rechazo de la técnica por el paciente, la infección en el sitio de la inyección, sepsis y otros estados de insuficiencia circulatoria, diagnóstico de hipertensión endocraneana y la existencia de coagulopatía severa. El éxito de esta técnica depende en parte de la correcta elección del anestésico local; lo más comúnmente utilizados son lidocaína y bupivacaína.

\section{Key words:}

Spinal anasthesia, subarachnoid space, cerebrospinal fluid, local anasthesic

\section{Palabras clave:}

Anestesia espinal, espacio subaracnoideo, líquido cefalorraquídeo, anestésico local

\section{División de Anestesiología, Escuela de Medicina, Pontificia Universidad Católica de Chile. Santiago, Chile.}

Escuela de Medicina, Pontificia Universidad Católica de Chile. Santiago, Chile.

Fecha de recepción: 15 de octubre de 2020

Fecha de aceptación: 25 de octubre de 2020

\section{ORCID}

0000-0002-0464-8643

No se obtuvieron fuentes de financiamiento para el estudio.

Los autores no declaran tener conflictos de interés.

Correspondencia:

Fernando Altermatt Couratier

falterma@uc.cl 


\section{Introducción}

E n esta segunda monografía dedicada a los bloqueos espinales, nos concentraremos en describir dos aspectos relevantes para su realización: haremos una reseña de los conocimientos de anatomía que son importantes para esta técnica, y revisaremos las indicaciones y contraindicaciones para la realización de bloqueos espinales. Finalmente, describiremos las drogas más usadas en este procedimiento.

\section{Anatomía}

El bloqueo espinal, como todas las técnicas de anestesia regional requiere del conocimiento acabado de la anatomía; en este caso, de la columna vertebral y sus componentes.

La columna vertebral posee tres curvas en su extensión longitudinal: lordosis cervical y lumbar, así como una cifosis dorsal.

Una vértebra tipo estará compuesta por un cuerpo vertebral, desde donde salen dos ramas divergentes a posterior, que constituyen los pedículos, dando lugar a las apófisis transversas, desde donde a su vez, confluyen hacia la línea media las láminas, que se juntan para formar la apófisis espinosa (Figura 1).

Los componentes óseos de la columna están a su vez estabilizados entre sí por ligamentos. El ligamento supraespinoso conecta la punta de las apófisis espinosas desde C7 al sacro. Desde C7 hasta el cráneo este ligamento es estructuralmente distinto y se llama ligamento nucal. El ligamento interespinoso conecta los cuerpos de las apófisis espinosas. El ligamento amarillo conecta las láminas vertebrales de vértebras adyacentes. Los ligamentos longitudinales anterior y posterior conectan los cuerpos vertebrales por sus caras anterior y posterior.

El espacio peridural es un espacio virtual entre la cara interna del canal medular y la cara externa del

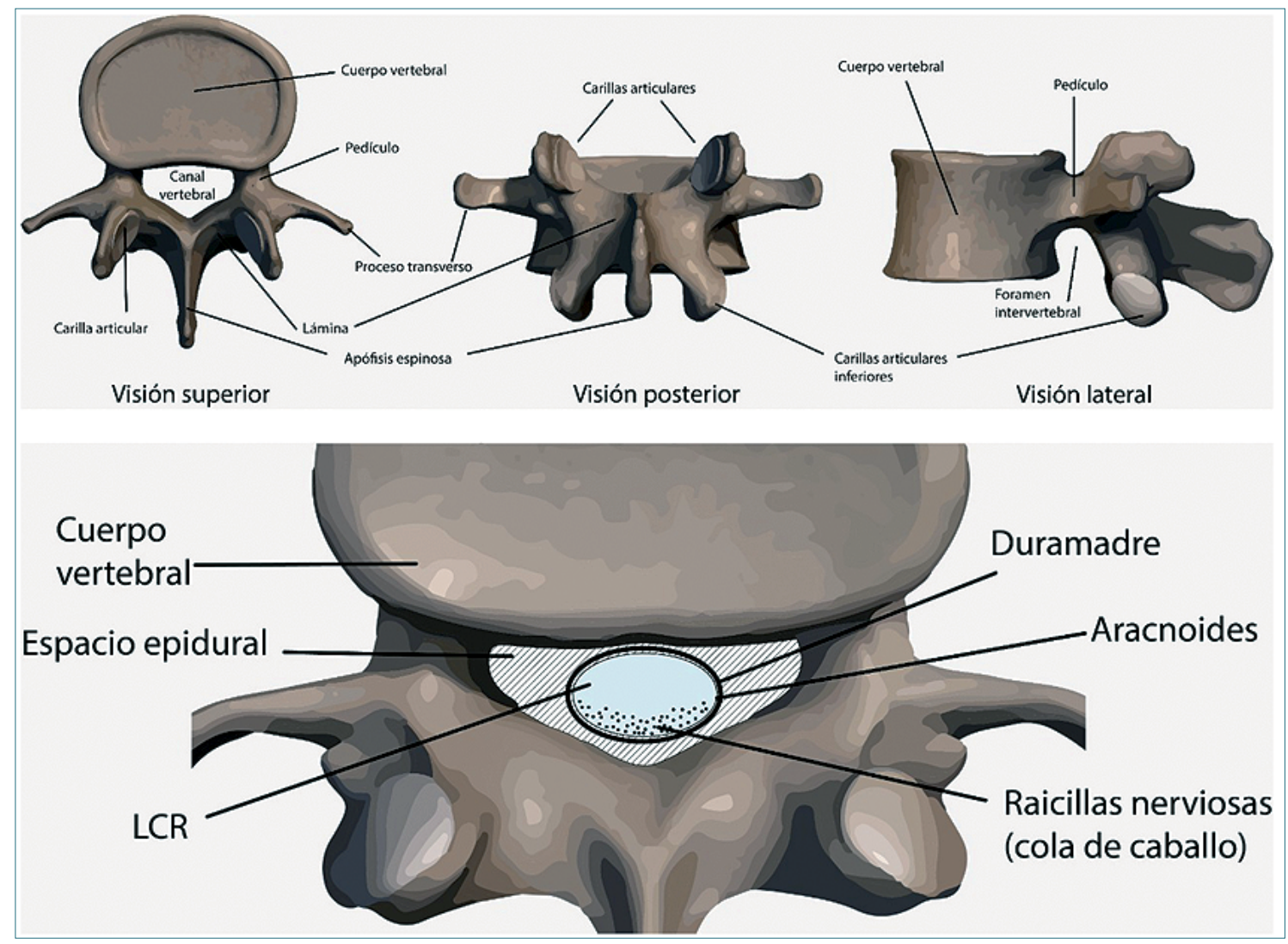

Figura 1. En la imagen superior, se ilustra una vertebra L2 en sus visones superior, posterior y lateral. En la imagen inferior, se ilustra una vertebra L5 en un paciente en decúbito supino. Las raicillas nerviosas que transcurren por el espacio subaracnoideo caen hacia posterior al estar el paciente en posición decúbito supino. 
saco dural. Las raíces de los nervios espinales atraviesan el espacio peridural en su trayecto hacia los forámenes intervertebrales. El espacio peridural está compuesto por grasa, tejido fibro-aerolar y plexos venosos. Los niveles más caudales del espacio peridural contienen proporcionalmente más grasa[1], en tanto que en los segmentos más cefálicos, en grandes áreas la duramadre contacta el hueso y los ligamentos del canal medular, sin estar unidos[2].

La médula espinal está rodeada en toda su extensión por estructuras óseas que le dan sostén y protección. El saco dural que contiene a la médula, se extiende hasta S2 y en ella se reconocen tres membranas: duramadre, aracnoides y piamadre.

La duramadre es una lámina de tejido conectivo que se extiende desde el foramen magno hasta S2. Tiene una forma oval en su sección transversal[1]. Su espesor no es homogéneo, siendo más gruesa en la línea media dorsal[3]. Está compuesta por fibras dispuestas en forma circunferencial y longitudinal hechas de colágeno y elastina, por lo que tiene una consistencia firme pero distensible. A pesar de su grosor, es permeable[4].

La aracnoides es una membrana delgada, avascular, estrechamente adosada a la duramadre por su superficie interna. Es altamente impermeable, lo que permite mantener el líquido cefalorraquídeo en el saco dural. La aracnoides es responsable del $90 \%$ de la resistencia a la migración de las drogas desde y hacia el liquido cefalorraquídeo[5].

La piamadre, la más interna de las tres membranas, es altamente vascularizada. Se encuentra adosada a la médula espinal. Distalmente termina en la forma del filum terminale, que ancla la médula al sacro.

Entre la aracnoides y la piamadre se encuentra el espacio subaracnoideo, en donde circula el líquido cefalorraquídeo y las raicillas nerviosas (Figura 1). El espacio subaracnoídeo posterior, aquel al cual normalmente se accede con la técnica espinal, contiene múltiples subdivisiones membranosas[6] (Figura 2).

Como se ilustra en la Figura 4, en posición fetal las raicillas nerviosas contenidas en el espacio subaracnoideo se van hacia anterior[7]. Es relevante ya que otorga un margen de seguridad adicional al momento de la punción lumbar (Figura 3).

La longitud de la médula espinal y por ende la extensión dentro del canal, depende de la edad de los sujetos: al nacer, se extiende aproximadamente hasta L3. En $51 \%$ de los adultos se extiende hasta L1, aunque en un tercio de los casos, llega hasta T12 y en $12 \%$, hasta L3 (Figura 4). Estudios usando resonancia nuclear magnética (RMN) reportan 19\% de sujetos en los que la médula llega bajo L1[8].
Estos datos anatómicos son muy relevantes para una técnica segura, dado que el trauma que puede causar una punción y posterior inyección intramedular puede tener consecuencias catastróficas. Abordajes por sobre L3 pueden asociarse a punciones del cono medular, con daño neurológico[9].

Wall et al., demostraron que una red dependiente de la aracnoides mantiene unidas las raíces a nivel del cono medular[10].

\section{Líquido cefalorraquídeo (LCR)}

El LCR es un ultrafiltrado del plasma, y es producido por los plexos coroídeos de los ventrículos laterales, tercer y cuarto ventrículo.

El volumen de LCR en un adulto es alrededor de $2 \mathrm{ml} / \mathrm{kg}$ y cerca de $0,5 \mathrm{~L}$ son producidos cada día[11]. Sus propiedades físico-químicas se enumeran en la Tabla 1.

La cisterna lumbar corresponde a la porción más amplia del espacio subaracnoídeo, distal a la médula espinal. Ésta alberga la cauda equina, constituida por raíces ventrales y dorsales de los nervios espinales $L 2$ a coccígeo 1, distribuidos alrededor del filum terminale. Es en esta zona donde frecuentemente se realiza la técnica espinal, ya que se aleja de la médula y da la mejor probabilidad de puncionar el saco dural.

\section{Indicaciones}

En términos generales el uso de anestesia espinal está indicado cuando el procedimiento quirúrgico puede lograrse con un nivel sensitivo de anestesia que no afecte el bienestar del paciente.

Las especialidades quirúrgicas en las cuales su uso se ha difundido son: cirugía ortopédica de extremidades inferiores, cirugía general infraumbilical, genitourinaria, ginecológica y obstétrica.

\section{Contraindicaciones}

Como en todo procedimiento médico, la anestesia espinal tiene contraindicaciones absolutas y relativas. Contraindicaciones absolutas son el rechazo de la técnica por el paciente, la infección en el sitio de la inyección, sepsis y otros estados de insuficiencia circulatoria, diagnóstico de hipertensión endocraneana y la existencia de coagulopatía severa (Tabla 2). La justificación de esta última es el riesgo de aparición de un hematoma peridural con compresión medular y daño neurológico subsiguiente. La hipertensión en- 


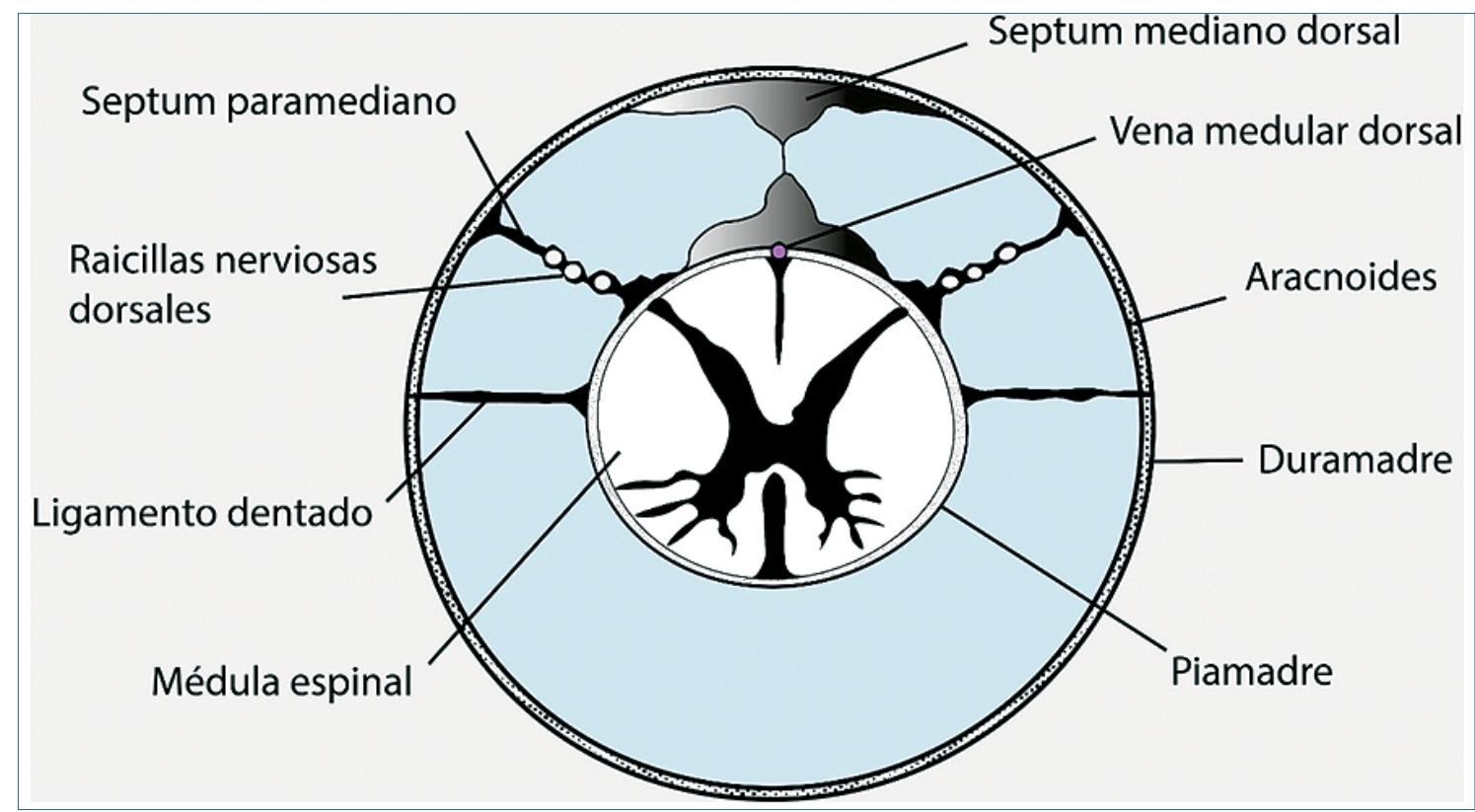

Figura 3. En posición fetal, las raicillas nerviosas se van hacia anterior, otorgando un margen de seguridad adicional al momento de la punción lumbar. Adaptado de Takiguchi T, Yamaguchi S, Hashizume Y, Kitajima T. Movement of the Cauda Equina during the lateral decubitus position with fully flexed leg. Anesthesiology. 2004;101(5):1250.

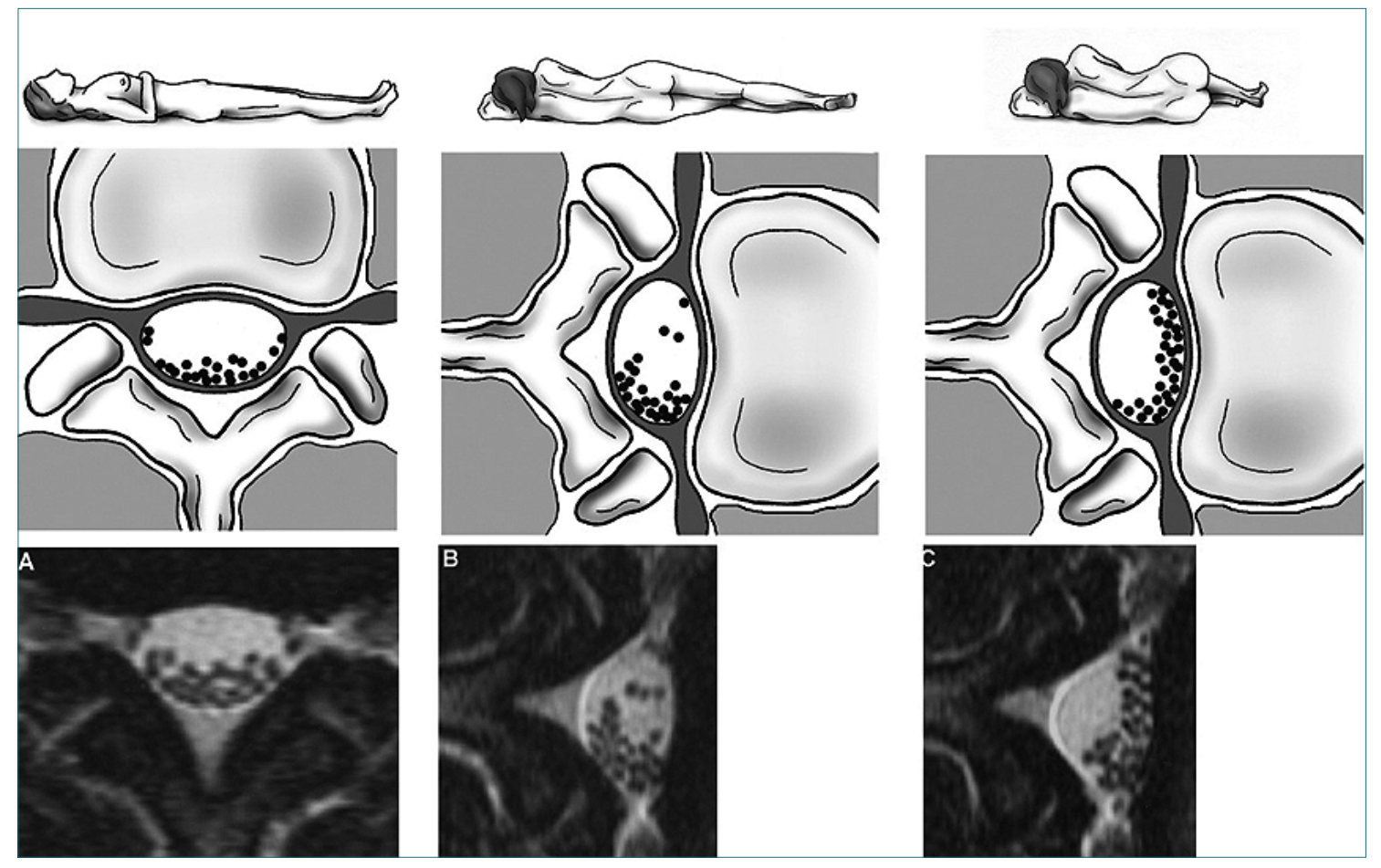

Figura 4. La médula espinal en $51 \%$ de los adultos se extiende hasta L1, aunque en un tercio de los casos, llega hasta T12 y en $12 \%$, hasta L3 (42). 


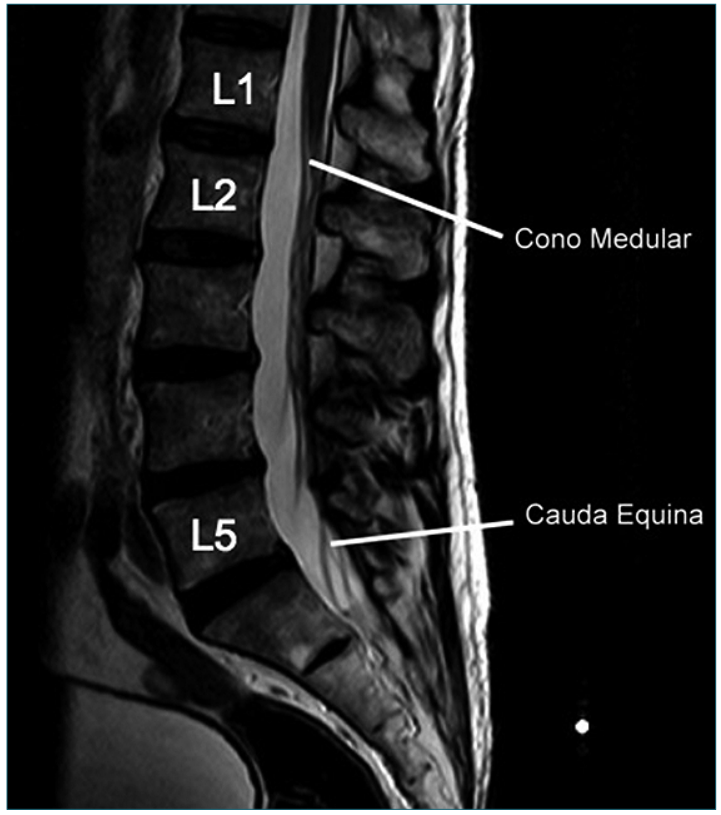

Figura 5. Cono medular y cauda equina. Resonancia magnética fase $\mathrm{T} 2$, corte sagital.

docraneana es una contraindicación debido al riesgo de pérdida de líquido cefalorraquídeo, y la posibilidad de una herniación cerebral descendente.

La sepsis es un cuadro generalizado grave en respuesta a una infección, con hipoperfusión tisular. Estos pacientes se caracterizan por compromiso hemodinámico, muchas veces requirente de drogas vasoactivas. En este contexto no parece apropiado usar una técnica que conlleva un bloqueo simpático y mayor detrimento de la función cardiovascular.

Contraindicaciones relativas pueden ser la existencia de un foco de infección, distinto del sitio de punción. La posibilidad de efectuar una punción dural durante una bacteremia podría favorecer el desarrollo de una meningitis[12]. Otras posibles contraindicaciones son enfermedad neurológica no definida o daño neurológico en evolución y pacientes profundamente hipovolémicos.

Finalmente, la indicación de usar o no la técnica debe basarse en un juicio médico tomando en cuenta sus ventajas y desventajas. Entre sus desventajas se encuentra la presencia de fallas anestésicas. La falla puede ser inicial por falta de nivel o falta de intensidad del bloqueo. La tasa de fracaso probablemente alcance menos del 1\%[13]. Existe la posibilidad que la cirugía se prolongue en el tiempo y la anestesia se vuelva insuficiente. Otras desventajas son la ansiedad frente al procedimiento, la necesidad de experiencia
Tabla 1. Propiedades físico-químicas el líquido cefalorraquídeo

\author{
Propiedades físico-químicas del líquido cefalorraquí- \\ deo \\ Aspecto: Cristalino \\ Osmolaridad: 292-7 mOsm•|t- ${ }^{1}$ \\ Densidad: $1.00050-1.00070 \mathrm{~g} \bullet \mathrm{ml}^{-1}$ \\ Electrolitos y glucosa = plasma \\ $\mathrm{pCO}_{2}: 48 \mathrm{mmHg}$ \\ $\mathrm{pH}: 7,32$ \\ Celularidad: $<5$ por $\mathrm{ml}$ \\ Proteínas: $20-40$ mg\% \\ Presión (lumbar): $60-80 \mathrm{mmH}_{2} \mathrm{O}$ \\ Volumen: $150 \mathrm{ml}$
}

Adaptado de Manual de Anestesiología, Concha M. de la Cuadra JC. Ediciones Universidad Católica de Chile, Santiago 2006.

Tabla 2. Contraindicaciones absolutas de la anestesia espinal

\section{Contraindicaciones absolutas de anestesia espinal}

Rechazo a la técnica

Trastorno grave de la coagulación

Infección sitio de punción

Sepsis

Hipertensión endocraneana

del operador, una mayor latencia al compararlo con la anestesia general, imposibilidad de usarla en todo tipo de cirugías, potenciales efectos colaterales derivados de las drogas usadas y riesgo de complicaciones neurológicas permanentes.

\section{Drogas}

El éxito de la anestesia espinal depende, entre otros factores, de la correcta elección del anestésico local y de comprender sus características farmacológicas. Los anestésicos locales (AL) son drogas capaces de producir bloqueo reversible de la conducción nerviosa. Químicamente, los anestésicos locales son bases débiles, constituidas por un anillo aromático unido a un grupo amino por un enlace amida o éster, dividiéndose así en dos grupos: AL amino-amidas y 


\begin{tabular}{lccc}
\hline \multicolumn{1}{c}{ Tabla 3. Dosis y duración de anestésicos locales en anestesia espinal } \\
\hline \multicolumn{1}{c}{ Anestesico local } & Dosis & Altura de bloqueo & $\begin{array}{c}\text { Tiempo de regresión } 2 \\
\text { segmentos (min) }\end{array}$ \\
Lidocaina isobárica & $40 \mathrm{mg}$ & $\mathrm{T} 12$ & 44 \\
Lidocaina isobárica & $80 \mathrm{mg}$ & $\mathrm{T} 4$ & 33 \\
Lidocaina hiperbárica & $60 \mathrm{mg}$ & $\mathrm{T} 3$ & 48 \\
Mepivacaina isobárica & $60 \mathrm{mg}$ & $\mathrm{T} 4$ & 95 \\
Bupivacaina isobárica & $10 \mathrm{mg}$ & $\mathrm{T}$ & 33 \\
Bupivacaina isobárica & $17,5 \mathrm{mg}$ & $\mathrm{T} 8$ & 155 \\
Bupivacaina hiperbárica & $8 \mathrm{mg}$ & $\mathrm{T} 5$ & 59 \\
Bupivacaina hiperbárica & $15 \mathrm{mg}$ & $\mathrm{T} 4$ & 110 \\
\hline
\end{tabular}

Adaptado de Wong, C. A. (2007). Spinal and epidural anesthesia. New York, McGraw Hill Medical.

AL amino-ésteres. La lidocaína y la bupivacaína, los anestésicos más usados en nuestro medio son de la familia de las amino amidas.

El mecanismo de acción de estas drogas es el bloqueo de los canales de sodio voltaje dependiente en la membrana celular de los axones, impidiendo alcanzar el potencial umbral para la despolarización. Según la densidad que las soluciones de los fármacos utilizados tengan en relación a la densidad del LCR, habrá soluciones igualmente, más o menos densas que él. Esta comparación de su densidad respecto al LCR se denomina baricidad. Es específica para una temperatura determinada. Esto es importante, puesto que la densidad relevante es aquella que la droga tendrá a temperatura corporal (alrededor de $37^{\circ} \mathrm{C}$ ).

Las drogas podrán ser entonces isobáricas, si es que su densidad es similar a la del LCR, hipobáricas, si es que es menos densa que el LCR, e hiperbáricas, si es que es más densa que éste.

Los cambios en la baricidad normalmente se consiguen agregando a la solución ya sea agua destilada (para hacerla hipobárica), o dextrosa (para hacerla hiperbárica).

La gran mayoría de las soluciones de anestésicos locales tienen densidades semejantes a las del LCR a temperatura ambiente, pero a temperatura corporal se comportan como hipobáricas.

Por otro lado, la densidad del LCR puede variar, dependiendo de las condiciones de los pacientes, tales como el género, edad o embarazo[14].

Las propiedades clínicas particulares determinan la elección de una u otra. Estas se refieren principalmente a latencia, tiempo en que demora el $\mathrm{AL}$ en hacer efecto; duración del efecto anestésico y de la anestesia quirúrgica prevista, y potencia, cantidad de anestésico necesaria para producir una respuesta definida.

\section{Tetracaína}

Es un anestésico local de la familia de los aminoésteres. Se caracteriza por su duración intermedia a larga, y ha sido usado en soluciones iso-, hipo- e hiperbáricas. Su latencia es de 3-5 minutos y una duración de 70-180 minutos. Fue el primer anestésico de larga duración usado en anestesia espinal

\section{Lidocaína}

Ha sido el anestésico local de duración corta más usado en la anestesia espinal. Tiene una latencia de 2 a 5 minutos, con una duración de 60 a 90 minutos. Dosis de 50 a $100 \mathrm{mg}$ son útiles para cirugías de alrededor de una hora, pudiéndose disminuir la duración al disminuir las dosis[15].

Su uso se ha asociado a la aparición de Síntomas Neurológicos Transitorios (TNS en su sigla en inglés), manifestado como dolor lumbar bajo y disestesias irradiadas a la zona glútea, muslos y piernas[16]. Su uso al $5 \%$ se ha asociado a cuadros de síndrome de cauda equina, al ser administrada a través de microcatéteres espinales[17]. Estos efectos se han traducido en prácticamente un abandono del uso de lidocaína por vía subaracnoidea.

\section{2-Cloro-procaína (2-CP)}

La 2-CP es un derivado de la procaína. Ha estado disponible en el mercado desde más de 50 años. Inicialmente concebida como una alternativa rápida y más segura para la anestesia obstétrica[18] (es un amino-éster, por lo que es degradada por la pseudocolinesterasa, disminuyendo el riesgo de toxicidad sis- 
témica), cayó en descrédito al asociarse a cuadros de neurotoxicidad, con el uso de soluciones conteniendo bisulfito de sodio como antioxidante[19].

Una nueva formulación, libre de preservantes, ha demostrado tener un perfil que la hace ideal para su uso en cirugía ambulatoria: corta latencia, corta duración y una baja incidencia de TNS.

El uso de $50 \mathrm{mg}$ de 2-CP al 1\%, en una solución isobárica y compararla con la misma masa de lidocaína al $1 \%$ en artroscopia de rodilla ambulatoria ha demostrado una recuperación más rápida, que permitiría un alta más precoz[20]. Las experiencias de anestesia espinal con cloroprocaina han ido consolidando su uso en cirugía ambulatoria en reemplazo de lidocaína o bajas dosis de bupivacaina.

\section{Ropivacaína}

Ha sido usada tanto en sus formas iso- e hiperbárica. La forma hiperbárica se asocia a bloqueos más consistentes, de menor duración que la isobárica[21]. En su uso espinal, esto se traduce en tiempos de recuperación más cortos, si se compara con dosis similares de bupivacaína[22].

\section{Bupivacaína}

Es una amino amida de duración intermedia a larga. Tiene una latencia de 5 a 8 minutos y una duración de 90-150 minutos. Se usa tanto en formas iso- hipo- o hiperbáricas. Ajustando la dosis, pueden obtenerse anestesias de larga duración (10-20 mg de soluciones iso o hiperbáricas) o de corta duración (5-8 $\mathrm{mg})$, que la harían una buena alternativa ante la lidocaína, con una incidencia de TNS considerablemente menor[23].

\section{Coadyuvantes}

Al igual que en otras técnicas de anestesia regional, en el uso de anestesia espinal se pueden emplear otras drogas que aumenten el efecto o la duración de los bloqueos espinales alcanzados por el anestésico local e idealmente disminuir los efectos no deseados como el bloqueo motor prolongado y el bloqueo simpático muy acentuado. Algunos de los más frecuentemente utilizados son: vasoconstrictores, agonistas $\alpha$-adrenérgicos, opioides e inhibidores de la acetilcolinesterasa (AChE).

\section{Vasoconstrictores}

Prolongan e intensifican el efecto de los anestésicos locales en una forma dosis-dependiente[24], por la vasoconstricción tisular que producen, disminuyendo la absorción sistémica del anestésico local, manteniendo la droga en contacto con las terminales nerviosas.

La epinefrina se emplea en dosis de 100 a 200 $\mu \mathrm{g}[25]$, prolongando clínicamente el efecto del anestésico local.

La fenilefrina se usa en dosis de 2-5 mg, prolongando el efecto de lidocaína espinal[26].

Aún cuando el uso de vasoconstrictores no comprometa el flujo sanguíneo de la médula[27], existe evidencia que al menos el uso de fenilefrina en asociación con tetracaína contribuye al desarrollo de TNS[28], efecto que no ha sido observado con epinefrina[29].

\section{Agonistas $\alpha-2$ adrenérgicos}

El uso de estas drogas persigue aumentar la analgesia y prolongar el bloqueo sensitivo y motor derivado del anestésico local. El efecto sobre el bloqueo sensitivo tendría su causa en mecanismos de modulación pre y post-sináptica en el asta dorsal de la médula[30]. El efecto sobre el bloqueo motor sería mediado por la hiperpolarización neuronal a nivel del asta ventral, potenciando la acción de los anestésicos locales.

La adición de clonidina en dosis de $150 \mu \mathrm{g}$ agregados a una solución de bupivacaína, prolonga el bloqueo sensitivo y motor secundarios a la anestesia espinal en pacientes sometidos a cirugía ortopédica[31]. Su uso en dosis más altas (300-600 $\mu \mathrm{g})$, se asocia a efectos indeseados, como hipotensión arterial o sedación excesiva[32].

El uso de dexmedetomidina intratecal prolonga el bloqueo espinal significativamente, tanto sensitivo como motor. Comparado con fentanilo como coadyuvante, la prolongación es mayor, con menos prurito y sin diferencia en hipotensión arterial y bradicardia.

\section{Opioides}

La administración intratecal de opioides produce un bloqueo selectivo de las aferencias dolorosas conducidas por fibras $A \delta$ y $C$, sin tener efectos significativos sobre las raíces dorsales o potenciales de acción somatosensitivos[33]. Sin embargo, su uso se asocia a efectos colaterales, donde el más frecuente es prurito, seguido por náuseas, vómitos y riesgo de depresión respiratoria, que es similar a otras vías de administración, en forma dosis dependiente[34].

Drogas más hidrofílicas, como la morfina, producen una analgesia espinal de excelente calidad y larga duración, con una latencia larga, duración prolongada y riesgo de depresión respiratoria tardío, producto de su ascenso rostral a través del LCR[35]. Dosis de entre 50- $200 \mu \mathrm{g}$ son las más usadas para balancear un adecuado efecto analgésico con un menor riesgo 
de complicaciones derivadas de su uso[36].

Drogas más lipofílicas, como el fentanyl o sufentanil, tienen una latencia y una duración de la analgesia relativamente más corta, si se le compara con la morfina[33]. Dosis de 10-20 $\mu \mathrm{g}$ de fentanyl o 1-10 $\mu \mathrm{g}$ de sufentanil son comúnmente usados para potenciar el efecto analgésico, prolongando el bloqueo sensitivo pero no la duración del bloqueo motor obtenido por los anestésicos locales[37].

\section{Inhibidores de la Acetilcolinesterasa (AChE)}

Estas drogas impiden la degradación de acetilcolina (ACh) y producen analgesia cuando son administradas por vía intratecal. El efecto anti-nociceptivo de la neostigmina se debe a la activación de receptores muscarínicos M1 y M2 en la médula espinal, aumento de la ACh circulante y la producción de óxido nítrico (NO)[38].

La administración de neostigmina en dosis de 25 a $100 \mu \mathrm{g}$ agregados a una solución de bupivacaína se asocian a disminución de los requerimientos analgésicos posquirúrgicos, pero con una alta incidencia de náuseas y vómitos.

\section{Determinantes de la duración}

Discutir sobre la duración de la anestesia espinal es a veces complejo y requiere de ciertas definiciones operacionales. Dado que el bloqueo espinal regresa desde los segmentos más altos bloqueados, normalmente se usa la regresión de dos o cuatro segmentos como una medida de la duración de la anestesia.

De eso se desprende que la duración de la acción de un bloqueo determinado dependerá de los dermatomas involucrados en la cirugía. Así, si el nivel alcanzado fue de T4 y la cirugía compromete territorios lumbares bajos y sacros, la duración útil como anestesia quirúrgica, será considerablemente mayor que si la cirugía fuese abdominal.
La elección de la droga a usar es probablemente el mayor determinante de la duración de la anestesia resultante: drogas con mayor afinidad por proteínas, como la tetracaína o bupivacaína, tienen una duración mayor que aquellas con menos afinidad por proteínas, como la lidocaína, mepivacaína o procaína.

Para una misma droga, la duración de la anestesia espinal depende primariamente de la masa de droga administrada. A mayores dosis se obtienen duraciones más prolongadas.

Por otro lado, ni el volumen ni la concentración de la solución administrada tienen efecto sobre la duración de la anestesia espinal, si la masa de droga se mantiene constante[39]. Diferente es para la analgesia, donde el volumen y la concentración tienen un efecto relevante[40].

La densidad de la solución, por su parte, tiene efecto sobre la duración de la anestesia espinal: las drogas hipobáricas tienen una duración más prolongada, seguidas de las isobáricas y finalmente las hiperbáricas[41].

La extensión del bloqueo obtenido también tiene efecto sobre la duración del bloqueo. A mayor extensión del bloqueo para una droga determinada, menor duración del bloqueo. Una droga limitada a un área pequeña durará más, puesto que la concentración alrededor del nervio será mayor. Del mismo modo, bloqueos más altos regresarán más rápido, dado que las concentraciones resultantes a cefálico son relativamente menores, por lo que la caída de éstas bajo el umbral de la concentración mínima efectiva será antes.

En conclusión, en esta revisión hemos descrito elementos de anatomía que son importantes para entender cómo funciona y cómo se administra la anestesia espinal. Del mismo modo, hemos enumerado las indicaciones y contraindicaciones para esta técnica y finalmente, hemos hecho una descripción de las drogas más comúnmente utilizadas, y sus efectos en la calidad de los bloqueos, en su extensión y duración.

\section{Referencias}

1. Hogan QH. Lumbar epidural anatomy. A new look by cryomicrotome section. Anesthesiology. 1991;75(5):767-75. https:// doi.org/10.1097/00000542199111000-00007

2. Hogan Q. Anatomy of spinal anesthesia: some old and new findings. Reg Anesth Pain Med. 1998;23(4):3403; discussion 84-7. https:// doi.org/10.1097/00115550199823040-00003

3. Cheng PA. The anatomical and clinical aspects of epidural anesthesia. I. Anesth Analg.
1963;42:398-406. https:// doi.org/10.1213/00000539196305000-00023

4. Fink BR, Walker S. Orientation of fibers in human dorsal lumbar dura mater in relation to lumbar puncture. Anesth Analg. 1989;69(6):768-72. https:// doi.org/10.1213/00000539- 


\section{0-00014}

5. Shanthaveerappa TR, Bourne GH The 'perineural epithelium', a metabolically active, continuous, protoplasmic cell barrier surrounding peripheral nerve fasciculi. J Anat. 1962;96:527-37.

6. Nauta HJ, Dolan E, Yasargil MG. Microsurgical anatomy of spinal subarachnoid space. Surg Neurol. 1983;19(5):431-7. https://doi.org/10.1016/00903019(83)90141-6

7. Takiguchi T, Yamaguchi S, Hashizume Y, Kitajima T. Movement of the Cauda Equina during the lateral decubitus position with fully flexed leg. Anesthesiology. 2004;101(5):1250. https:// doi.org/10.1097/00000542200411000-00044

8. Broadbent $C R$, Maxwell WB, Ferrie R, Wilson DJ, Gawne-Cain M, Russell R. Ability of anaesthetists to identify a marked lumbar interspace. Anaesthesia. 2000;55(11):1122-6. https:// doi.org/10.1046/j.13652044.2000.01547-4.x

9. Reynolds F. Logic in the safe practice of spinal anaesthesia. Anaesthesia. 2000;55(11):10456. https://doi.org/10.1046/ j.1365-2044.2000.01830.x

10. Wall EJ, Cohen MS, Abitbol JJ, Garfin SR. Organization of intrathecal nerve roots at the level of the conus meduIlaris. J Bone Joint Surg Am. 1990;72(10):1495-9. https:// doi.org/10.2106/00004623199072100-00010

11. Cutler RW, Page L, Galicich J, Watters GV. Formation and absorption of cerebrospinal fluid in man. Brain. 1968;91(4):70720. https://doi.org/10.1093/ brain/91.4.707

12. Carp H, Bailey S. The association between meningitis and dural puncture in bacteremic rats. Anesthesiology. 1992;76(5):739-42. https:// doi.org/10.1097/00000542199205000-00012

13. Fettes PD, Jansson JR, Wildsmith $J A$. Failed spinal anaesthesia: mechanisms, management, and prevention. $\mathrm{Br} J$ Anaesth. 2009;102(6):739-48. https://doi. org/10.1093/bja/aep096

14. Lui A, Polis T, Cicutti N. Densities of cerebrospinal fluid and spinal anaesthetic solutions in surgical patients at body temperature. Canadian Journal of Anesthesia / Journal canadien d'anesthésie. 1998;45(4):297-303. https://doi. org/10.1007/BF03012018

15. Urmey WF, Stanton J, Peterson $M$, Sharrock NE. Combined spinal-epidural anesthesia for outpatient surgery. Dose-response characteristics of intrathecal isobaric lidocaine using a 27-gauge Whitacre spinal needle. Anesthesiology. 1995;83(3):528-34. https:// doi.org/10.1097/00000542199509000-00011

16. Pollock JE. Neurotoxicity of intrathecal local anaesthetics and transient neurological symptoms. Best Pract Res Clin Anaesthesiol. 2003;17(3):47184. https://doi.org/10.1016/ S1521-6896(02)00113-1

17. Rigler ML, Drasner K, Krejcie TC, Yelich SJ, Scholnick FT, DeFontes J, et al. Cauda equina syndrome after continuous spinal anesthesia. Anesth Analg. 1991;72(3):275-81. https:// doi.org/10.1213/00000539199103000-00001

18. Foldes FF, Mc NP. 2-Chloroprocaine: a new local anesthetic agent. Anesthesiology. 1952;13(3):287-96. https:// doi.org/10.1097/00000542195205000-00009

19. avindran RS, Bond VK, Tasch MD, Gupta CD, Luerssen TG. Prolonged neural blockade following regional analgesia with 2-chloroprocaine. Anesth Analg.
1980;59(6):447-51. https:// doi.org/10.1213/00000539198006000-00013

20. Casati A, Fanelli G, Danelli G, Berti M, Ghisi D, Brivio M, et al. Spinal anesthesia with lidocaine or preservative-free 2-chlorprocaine for outpatient knee arthroscopy: a prospective, randomized, double-blind comparison. Anesth Analg. 2007;104(4):95964. https://doi.org/10.1213/01. ane.0000258766.73612.d8

21. Khaw KS, Ngan Kee WD, Wong $\mathrm{M}, \mathrm{Ng} F$, Lee A. Spinal ropivacaine for cesarean delivery: a comparison of hyperbaric and plain solutions. Anesth Analg. 2002;94(3):680-5; table of contents. https:// doi.org/10.1097/00000539200203000-00037

22. Gautier PE, De Kock M, Van Steenberge A, Poth N, LahayeGoffart B, Fanard L, et al. Intrathecal ropivacaine for ambulatory surgery. Anesthesiology. 1999;91(5):1239-45. https:// doi.org/10.1097/00000542199911000-00013

23. Hiller A, Rosenberg PH. Transient neurological symptoms after spinal anaesthesia with $4 \%$ mepivacaine and $0.5 \%$ bupivacaine. Br J Anaesth. 1997;79(3):301-5. https://doi. org/10.1093/bja/79.3.301

24. Kristensen JD, Karlsten R, Gordh T. Spinal cord blood flow after intrathecal injection of ropivacaine and bupivacaine with or without epinephrine in rats. Acta Anaesthesiol Scand. 1998;42(6):685-90. https://doi. org/10.1111/j.1399-6576.1998. tb05302.x

25. Kito K, Kato H, Shibata M, Adachi T, Nakao S, Mori K. The effect of varied doses of epinephrine on duration of lidocaine spinal anesthesia in the thoracic and lumbosacral dermatomes. Anesth Analg. 
1998;86(5):1018-22. https:// doi.org/10.1213/00000539199805000-00021

26. Vaida GT, Moss P, Capan LM, Turndorf $\mathrm{H}$. Prolongation of lidocaine spinal anesthesia with phenylephrine. Anesth Analg. 1986;65(7):781-5. https:// doi.org/10.1213/00000539198607000-00014

27. Bahar M, Cole G, Rosen M, Vickers MD. Histopathology of the spinal cord after intrathecal cocaine, bupivacaine, lignocaine and adrenaline in the rat. Eur J Anaesthesiol. 1984;1(3):293-7.

28. Sakura S, Sumi M, Sakaguchi $Y$, Saito $Y$, Kosaka $Y$, Drasner K. The addition of phenylephrine contributes to the development of transient neurologic symptoms after spinal anesthesia with $0.5 \%$ tetracaine. Anesthesiology. 1997;87(4):771-8. https:// doi.org/10.1097/00000542199710000-00009

29. Freedman JM, Li DK, Drasner K, Jaskela MC, Larsen B, Wi S. Transient neurologic symptoms after spinal anesthesia: an epidemiologic study of 1,863 patients. Anesthesiology. 1998;89(3):633-41. https:// doi.org/10.1097/00000542199809000-00012

30. Eisenach JC, De Kock M, Klimscha W. alpha(2)-adrenergic agonists for regional anesthesia. A clinical review of clonidine (1984-1995). Anesthesiology. 1996;85(3):655-74. https:// doi.org/10.1097/00000542199609000-00026

31. Strebel S, Gurzeler JA, Schneider MC, Aeschbach A, Kindler $\mathrm{CH}$. Small-dose intrathecal clonidine and isobaric bupivacaine for orthopedic surgery: a dose-response study. Anesth Analg. 2004;99(4):1231-8, table of contents. https:// doi.org/10.1213/01. ANE.0000133580.54026.65

32. Niemi L. Effects of intrathecal clonidine on duration of bupivacaine spinal anaesthesia, haemodynamics, and postoperative analgesia in patients undergoing knee arthroscopy. Acta Anaesthesiol Scand. 1994;38(7):724-8. https://doi. org/10.1111/j.1399-6576.1994. tb03985.x

33. Hamber EA, Viscomi CM. Intrathecal lipophilic opioids as adjuncts to surgical spinal anesthesia. Reg Anesth Pain Med. 1999;24(3):255-63. https:// doi.org/10.1097/00115550199924030-00015

34. Gustafsson LL, Schildt B, Jacobsen K. Adverse effects of extradural and intrathecal opiates: report of a nationwide survey in Sweden. Br J Anaesth. 1982;54(5):479-86. https://doi. org/10.1093/bja/54.5.479

35. Ummenhofer WC, Arends $\mathrm{RH}$, Shen DD, Bernards CM. Comparative spinal distribution and clearance kinetics of intrathecally administered morphine, fentanyl, alfentanil, and sufentanil. Anesthesiology. 2000;92(3):739-53. https:// doi.org/10.1097/00000542200003000-00018

36. Slappendel R, Weber EW, Dirksen R, Gielen MJ, van Limbeek J. Optimization of the dose of intrathecal morphine in total hip surgery: a dose- finding study. Anesth Analg. 1999;88(4):822-6. https:// doi.org/10.1213/00000539199904000-00026

37. Patterson L, Avery N, Chan P, Parlow JL. The addition of fentanyl does not alter the extent of spread of intrathecal isobaric bupivacaine in clinical practice. Can J Anaesth. 2001;48(8):76872. https://doi.org/10.1007/ BF03016692

38. Chiari A, Eisenach JC. Spinal anesthesia: mechanisms, agents, methods, and safety. Reg Anesth Pain Med. 1998;23(4):357-62; discussion 84-7. https:// doi.org/10.1097/00115550199823040-00007

39. King HK, Wooten DJ. Effects of drug dose, volume, and concentration on spinal anesthesia with isobaric tetracaine. Reg Anesth. 1995;20(1):45-9.

40. Parpaglioni R, Frigo MG, Lemma A, Sebastiani M, Barbati G, Celleno D. Minimum local analgesic dose: effect of different volumes of intrathecal levobupivacaine in early labor. Anesthesiology. 2005;103(6):1233-7. https:// doi.org/10.1097/00000542200512000-00019

41. Concepcion MA. Spinal anesthetic agents. Int Anesthesiol Clin. 1989;27(1):21-5. https:// doi.org/10.1097/00004311198902710-00005

42. levins FA. Accuracy of placement of extradural needles in the L3-4 interspace: comparison of two methods of identifying $L 4$. Br J Anaesth. 1991;66(3):3812. https://doi.org/10.1093/ bja/66.3.381 